\title{
Ultrasound Combined with Microbubbles Enhances the Effects of Methylprednisolone in Lipopolysaccharide-Induced Human Mesangial Cells
}

\author{
Peng-Fei Sun, ${ }^{1}$ Tian Tian, ${ }^{1}$ Li-Na Chen, Rong-Guo Fu, Shan-Shan Xu, Hong Ai, Bing Wang, \\ Jian Zhang, Ruo-Yan Si, Zhonglin Chai, Mark E. Cooper, and Shu-Ting Ren \\ Department of Pathology (P.-F.S., T.T., B.W., J.Z., S.-T.R.), Department of Pharmacology (L.-N.C.), and Therapeutic Vaccines \\ Engineering Center of Shaanxi Province (B.W.), School of Basic Medical Sciences, Xi'an Jiaotong University Health Science \\ Center, Xi'an, People's Republic of China; Department of Pathology, Xi'an City Center Hospital, Xi'an, People's Republic of \\ China (P.-F.S.); Department of Nephrology, Second Affiliated Hospital of Xi'an Jiaotong University, Xi'an, People's Republic of \\ China (R.-G.F.); Department of Biomedical Engineering, School of Life Science and Technology, Xi'an Jiaotong University, Xi'an, \\ People's Republic of China (S.-S.X.); Department of Ultrasound, First Affiliated Hospital of Xi'an Jiaotong University, Xi'an, \\ People's Republic of China (H.A.); Shanghai Medical College, Fudan University, Shanghai, People's Republic of China (R.-Y.S.); \\ and Department of Diabetes, Central Clinical School, Monash University, Melbourne, Australia (Z.C., M.E.C.)
}

Received November 7, 2017; accepted March 9, 2018

\section{ABSTRACT}

A novel drug delivery system mediated by ultrasound (US) combined with microbubbles (MBs) (US+MB) could improve local drug concentration to enhance its efficacy. To investigate the influence of US+MB on methylprednisolone (MP), the effect of US+MB combined with MP (US+MB + MP) on lipopolysaccharide (LPS)-induced human mesangial cells (HMCs) and the underlying mechanism were explored in this study. The results revealed that HMCs treated with LPS underwent significant proliferation and exhibited an increase in nuclear transcription factor $-\kappa \mathrm{B}(\mathrm{NF}-\kappa \mathrm{B})$ and transforming growth factor- $\beta 1$ (TGF- $\beta 1$ ) expression and a decrease in cellular apoptosis. This effect was significantly inhibited by MP (30-100 $\mu \mathrm{g} / \mathrm{ml})$, US combined with
MBs $\left(3.22 \times 10^{7}\right.$ and $8.05 \times 10^{7}$ bubbles $\left./ \mathrm{ml}\right)$, and US combined with both MBs $\left(1.29 \times 10^{7}\right.$ bubbles $\left./ \mathrm{ml}\right)$ and MP $(12 \mu \mathrm{g} / \mathrm{ml})(\mathrm{US}+$ MB1 + MP12). The effect of US +MB1 + MP12 was better than the effect of $12 \mu \mathrm{g} / \mathrm{ml}$ of MP alone and was similar to the effect of $100 \mu \mathrm{g} / \mathrm{ml}$ of MP. Additionally, the intracellular free MP content was significantly higher in the US +MB1+MP12 group than in the MP12 group. US combined with MBs not only inhibited LPSinduced HMC proliferation and NF- $\kappa \mathrm{B}$ and TGF- $\beta 1$ expression and increased cellular apoptosis but also synergized with the pharmacologic effect of MP. The mechanism is partially due to the US-assisted MB local drug delivery and the antiinflammatory effect induced by US combined with MBs.

\section{Introduction}

As key glomerular cells that typically cover $30 \%$ of the glomerular capillaries, renal mesangial cells (RMCs) contribute to the regulation of glomerular filtration, the phagocytosis of immune complexes, and the production of the extracellular matrix (ECM) (Wu et al., 2011). However, RMCs also play an important role in the pathogenesis of several renal diseases (Radeke and Resch, 1992; Wu et al., 2011; Zhang et al., 2015). When activated by immunologic and inflammatory stimulation, RMCs not only proliferate and produce ECM but also generate cytokines and chemokines that influence the

This work was supported by the Scientific Technology Planning Foundation of Shaanxi Province (People's Republic of China, No. 2014K11-03-05-02 to S.-T.R.) and the Fundamental Research Funds for the Central Universities (People's Republic of China, No. xjj2015079 to S.-T.R.)

${ }^{1}$ P.-F.S. and T.T. contributed equally to this work.

https://.doi.org/10.1124/jpet.117.246223. progression of kidney disease (Sánchez-López et al., 2008). Abnormal RMC proliferation and ECM accumulation are generally characteristics of progressive kidney disease; both of these characteristics lead to glomerulosclerosis (Schöcklmann et al., 1999; Jamba et al., 2015). Thus, modulating the mitogenic activity of RMCs might be important for determining whether glomerular injury progresses to advanced ECM accumulation or recovers from transient RMC proliferation.

Glomerulonephritis is a group of renal diseases characterized by inflammation within the glomerulus, which is the major cause of end-stage renal disease worldwide. Although the mechanism is poorly understood, immune and inflammatory factors play an important role in the pathogenesis of glomerulonephritis (Imig and Ryan, 2013). Due to the powerful and effective anti-inflammatory and immunomodulatory activities, glucocorticoids (GCs) are the primary clinical therapy for several renal diseases with nephrotic syndromes.

ABBREVIATIONS: ECM, extracellular matrix; GAPDH, glyceraldehyde-3-phosphate dehydrogenase; GC, glucocorticoid; HMC, human mesangial cell; LPS, lipopolysaccharide; MB, microbubble; MP, methylprednisolone; MTT, 3-(4,5-dimethylthiazol-2-yl)-2,5-diphenyltetrazolium bromide; $\mathrm{NF}-\kappa \mathrm{B}$, nuclear transcription factor- $\kappa \mathrm{B}$; OD, optical density; PI, propidium iodide; RMC, renal mesangial cell; RP-HPLC, reverse-phase highperformance liquid chromatography; rt-PA, recombinant tissue plasminogen activator; TGF- $\beta 1$, transforming growth factor- $\beta 1$; US, ultrasound. 
Among the GCs, methylprednisolone (MP) is widely used in glomerulonephritis treatments due to its advantages of a strong anti-inflammatory effect and immune suppression. However, GCs have serious side effects and are clinically ineffective in $\sim 20 \%$ of children and $\sim 50 \%$ of adults (Ponticelli et al., 1999; MacHardy et al., 2009). Therefore, methods to enhance the efficacy but lessen the toxicity of GCs are currently a critical issue.

Ultrasound (US) combined with microbubbles (MBs) as the US contrast agent is a novel drug or gene delivery system that has been under development in many experimental studies (Anderson et al., 2016; Wang et al., 2016; Zhu et al., 2016). In this delivery system, the drugs and genes of interest are entrapped within MBs then delivered into the targeted region, or the drugs or genes penetrate into the targeted cells alongside MBs when they are coadministered under US irradiation; the delivery was primarily mediated by sonoporation (Ren et al., 2015; Li et al., 2016). The studies have shown that gene transfection efficiency or recombinant tissue plasminogen activator (rt-PA) thrombolysis activity is obviously enhanced by the coadministration of MBs and plasmid DNA or rt-PA under US exposure compared with the use of plasmid DNA or rt-PA alone through the local delivery of the gene or drug via sonoporation.

The effect of MB and MP coadministration mediated by US in glomerulonephritis therapy has been unclear. Lipopolysaccharide (LPS), a main component of the outer membrane of Gram-negative bacteria, is commonly used to induce inflammatory responses in mesangial cells in vitro. Our study explored the effect of US combined with MBs on MP in a LPS-induced human mesangial cells (HMCs) inflammatory response model and evaluated the underlying mechanism.

\section{Materials and Methods}

Cell Culture. The HMCs cell line, which was obtained from Professor Rong-Guo Fu (Department of Nephrology, Second Associated Hospital, School of Medicine, Xi'an Jiaotong University), was cultured in RPMI 1640 medium supplemented with $10 \%$ fetal bovine serum, penicillin $(100 \mathrm{U} / \mathrm{ml})$, and streptomycin $(100 \mathrm{mg} / \mathrm{ml}$ ) (all from Thermo Fisher Biochemical Products, Beijing, People's Republic of China) at $37^{\circ} \mathrm{C}$ in a humid incubator containing $5 \% \mathrm{CO}_{2}$ (Thermo Fisher Scientific, Waltham, MA).

Treatment. The HMCs were seeded into 96 -well or 12 -well plates (Corning, New York, NY). After 24 hours, the cells were treated with $10 \mu \mathrm{g} / \mathrm{ml}$ of LPS (Sigma-Aldrich, St. Louis, MO), as shown in Table 1, and simultaneously treated without (LPS treatment group) or with US (US group), with US combined with different MBs concentrations $\left(1.29 \times 10^{7}, 3.22 \times 10^{7}\right.$, or $8.05 \times 10^{7}$ bubbles $/ \mathrm{ml}$; US +MB1, US +MB2, and US+MB3 groups, respectively), with US combined with both MBs $\left(1.29 \times 10^{7}\right.$ bubbles $\left./ \mathrm{ml}\right)$ and MP $(12 \mu \mathrm{g} / \mathrm{ml}$, methylprednisolone sodium succinate for injection [Pfizer Manufacturing Belgium NV, Puurs, Belgium]; US+MB1+MP12 group), or with different concentrations of MP alone (12, 30, 75, and $100 \mu \mathrm{g} / \mathrm{ml}$; MP12, MP30, MP75, and MP100 groups, respectively) in the different experiment groups.

Based on the effect of different US parameters on LPS-induced HMC growth in vitro (data not shown), a frequency of $0.8 \mathrm{MHz}$ and an intensity of $2.79 \mathrm{~W} / \mathrm{cm}^{2}$ for 5 minutes at a $50 \%$ duty cycle was selected as the US parameters for the present study. We used a commercial therapeutic US device (ultrasound probe area: $2.8 \mathrm{~cm}^{2}$; Dongjian Company, Beijing, People's Republic of China). Briefly, the therapeutic US probe was immobilized in a 1-liter glass beaker, with the probe surface accessible through the center hole of a planar cover. The treated well then was placed on the surface of the US probe, and the coupling gel was used to keep them in close contact.
TABLE 1

The treatment in each experiment group

US parameters: $0.8 \mathrm{MHz}, 2.79 \mathrm{~W} / \mathrm{cm}^{2}, 5$ minutes, $50 \%$ cycle duty. -: without US treatment; +: with US treatment.

\begin{tabular}{|c|c|c|c|c|}
\hline Groups & LPS & MP & $\operatorname{MBs}\left(\times 10^{7}\right.$ bubbles $\left./ \mathrm{ml}\right)$ & US \\
\hline & \multicolumn{2}{|c|}{$\mu g / m l$} & & \\
\hline Control & 0 & 0 & 0 & - \\
\hline LPS treated & 10 & 0 & 0 & - \\
\hline MP12 & 10 & 12 & 0 & - \\
\hline MP30 & 10 & 30 & 0 & - \\
\hline MP75 & 10 & 75 & 0 & - \\
\hline MP100 & 10 & 100 & 0 & - \\
\hline US & 10 & 0 & 0 & + \\
\hline $\mathrm{US}+\mathrm{MB} 1$ & 10 & 0 & 1.29 & + \\
\hline $\mathrm{US}+\mathrm{MB} 2$ & 10 & 0 & 3.22 & + \\
\hline US+MB3 & 10 & 0 & 8.05 & + \\
\hline US+MB1+MP12 & 10 & 12 & 1.29 & + \\
\hline
\end{tabular}

SonoVue microbubble echo-contrast agent (Bracco SpA, Milan, Italy) was reconstituted in $5 \mathrm{ml}$ of sodium chloride $9 \mathrm{mg} / \mathrm{ml}(0.9 \%)$ solution, and the proper volume of SonoVue MBs solution then was added to the wells in the different experimental groups (three final concentrations of the MBs: $1.29 \times 10^{7}, 3.22 \times 10^{7}$, and $8.05 \times 10^{7}$ bubbles $\left./ \mathrm{ml}\right)$. Cells without any treatment were defined as the control. The cells were measured or harvested at 24 and 48 hours after treatment.

Cell Proliferation. Cell proliferation was measured using the 3-(4,5-dimethylthiazol-2-yl)-2,5-diphenyltetrazolium bromide (MTT) assay. Briefly, $20 \mu \mathrm{l}$ of the MTT reagent ( $5 \mathrm{mg} / \mathrm{ml}$; Sigma-Aldrich) was incubated with the cells for 4 hours at $37^{\circ} \mathrm{C}$ and subsequently removed. Then, $150 \mu \mathrm{l}$ of dimethylsulfoxide (Guangdong Guanghua Chemical Factor, Guanghua, People's Republic of China) was added to each well and agitated for 10 minutes. The optical density (OD) was measured at a wavelength of $490 \mathrm{~nm}$ on a microplate reader (Multiskan GO; Thermo Scientific).

The cell proliferation rate was calculated according to the following formula based on quadruplicate experiments: Cell proliferation rate $(\%)=$ $\left(\right.$ Mean $\mathrm{OD}_{\text {treatment group }}-$ Mean $\left.\mathrm{OD}_{\text {control group }}\right) /$ Mean $\mathrm{OD}_{\text {control group }} \times 100$.

Cell Apoptosis. Apoptosis was measured using the FITC Annexin V Apoptosis Detection Kit II (Beyotime Biotechnology, Shanghai, People's Republic of China) according to the manufacturer's instructions. The cells were harvested by trypsinization, centrifuged, and suspended with $1 \mathrm{ml}$ of cold phosphate-buffered saline. Then, $5 \mu \mathrm{l}$ of Annexin V-FITC was added and incubated for 15 minutes in the dark. Next, $10 \mu \mathrm{l}$ of propidium iodide (PI) was added to the cells and incubated for 5 minutes on ice in the dark.

Finally, the samples were analyzed using a flow cytometer (BD FACScan Flow Cytometer; Becton Dickinson, Franklin Lakes, NJ), and the results were analyzed with FACSDiva software 7.0 (Becton Dickinson). Analysis of the stained cells divided the cells into four groups as follows: viable (annexin $\mathrm{V}^{-} \mathrm{PI}^{-}$), early apoptotic (annexin $\mathrm{V}^{+} \mathrm{PI}^{-}$), late apoptotic (annexin $\mathrm{V}^{+} \mathrm{PI}^{+}$), and necrotic (annexin $\mathrm{V}^{-} \mathrm{PI}^{+}$) cells.

A minimum of 10,000 cells was collected per sample, and the apoptosis rate was calculated according to the following formula based on triplicate experiments: Apoptosis rate $(\%)=($ Early apoptotic cells percentage + Late apoptotic cells percentage $) \times 100$.

Semiquantitative Reverse Transcription Polymerase Chain Reaction Analysis. The cells were harvested and the total RNA extracted using the RNAiso Plus reagent (TaKaRa Biotechnology, Dalian, People's Republic of China) according to the manufacturer's instructions. We synthesized cDNA from $4 \mu \mathrm{g}$ of total RNA using the PrimeScript 1st Strand cDNA Synthesis Kit (TaKaRa Biotechnology, Dalian) according to the manufacturer's instructions.

Semiquantitative analysis of RNA expression was performed by PCR on a T100 Thermal Cycler (Bio-Rad Laboratories, Hercules, CA) compared with the control transcript (glyceraldehyde-3-phosphate dehydrogenase, GAPDH). The PCR protocol was as follows: initial denaturation $\left(95^{\circ} \mathrm{C}\right.$ for 3 minutes), followed by 30 cycles of denaturation $\left(94^{\circ} \mathrm{C}\right.$ for 30 seconds, $60^{\circ} \mathrm{C}$ for 30 seconds, and $72^{\circ} \mathrm{C}$ for 1 minute) and annealing and extension $\left(72^{\circ} \mathrm{C}\right.$ for 5 minutes). The specific 
oligonucleotide primers were designed by Takara TaKaRa Biotechnology, Dalian, and the primer sequences for the three genes-nuclear transcription factor- $\kappa \mathrm{B}(\mathrm{NF}-\kappa \mathrm{B})$, transforming growth factor- $\beta 1$ (TGF$\beta 1$ ), and GAPDH-are listed in Table 2. For visualizing the gene expression, we electrophoresed individual DNA fragments on a $2 \%(\mathrm{w} / \mathrm{v})$ agarose gel (Sigma-Aldrich) and treated them with ethidium bromide.

Western Blot Analysis. The cells were harvested using lysis buffer (Beyotime Biotechnology) at the indicated time points. The proteins were extracted, separated with SDS-PAGE, and transferred onto a nitrocellulose membrane. The membranes were blocked using Tris-buffered saline containing $0.1 \%$ Tween 20 and $5 \%$ nonfat milk for 1 hour. The protein samples were probed with a rabbit polyclonal antibody specific for NF- $\mathrm{B}$ (p65) (diluted 1:1500; Abcam, Cambridge, United Kingdom), a mouse monoclonal antibody specific for TGF- $\beta 1$ (diluted 1:500; Abcam), and GAPDH (diluted 1:5000; Boster, Wuhan, People's Republic of China) overnight at $4^{\circ} \mathrm{C}$. Subsequently, the membranes were incubated with a horseradish peroxidaseconjugated anti-rabbit or anti-mouse IgG (1:1000; Cell Signaling Technology, Boston, MA) for 1 hour at $25^{\circ} \mathrm{C}$. GAPDH served as the control. The protein levels were visualized using an enhanced chemiluminescence solution (Millipore, Billerica, MA), and the band intensity was quantified using ImageJ (U.S. National Institutes of Health, Bethesda, MD).

Intracellular MP Content Detection by Reverse-Phase High-Performance Liquid Chromatography. A total of $1.4 \times$ $10^{5}$ cells were seeded into a 12 -well plate and cultured for 24 hours. Then the cells were treated with $12 \mu \mathrm{g} / \mathrm{ml}$ of MP alone or $12 \mu \mathrm{g} / \mathrm{ml}$ of MP combined with US (using the US parameters described previously) and MBs at a final MB concentration of $1.29 \times 10^{7}$ bubbles $/ \mathrm{ml}$. At 24 and 48 hours after treatment, the cells were harvested by trypsinization, centrifuged, and suspended in $1 \mathrm{ml}$ of phosphatebuffered saline. The cell suspension was frozen at $-20^{\circ} \mathrm{C}$, thawed at room temperature 2 times and broken using a Sonifier cell disrupter (JY92-IIN; Ningbo Xinzhi Biotech, Ningbo, People's Republic of China).

After centrifugation, the supernatants were collected, and the intracellular MP content was detected by reverse-phase highperformance liquid chromatography (RP-HPLC) (Dalian Elite Analytical Instruments, Dalian, People's Republic of China). The area under the curve was integrated, and the MP concentration was calculated based on a linear calibration curve.

Statistical Analysis. All data are presented as the mean \pm S.E.M. Tukey's multiple comparison test was applied to compare cell proliferation, apoptosis, NF- $\kappa \mathrm{B}$ (p65) and TGF- $\beta 1$ expression, and the intracellular MP content between the groups. The analysis was performed using GraphPad Prism 5.0 (GraphPad Software, San Diego, CA). $P<0.05$ was considered statistically significant.

\section{Results}

Effect of Different Treatments on LPS-Induced HMC Proliferation. The effects of MP, US, and US combined with MBs with or without MP on LPS-induced HMC proliferation are presented in Fig. 1. The cell proliferation rate was significantly increased in the LPS treatment group compared with the control group at 24 and 48 hours after treatment $(P<$ 0.001). After MP treatment, the cell proliferation rates were significantly decreased in the MP30 (MP $30 \mu \mathrm{g} / \mathrm{ml}$ ), MP75 (MP $75 \mu \mathrm{g} / \mathrm{ml}$ ), and MP100 (MP $100 \mu \mathrm{g} / \mathrm{ml}$ ) groups at 24 hours
$(P<0.001,0.01$, or 0.05$)$ but were significantly decreased in all MP treatment groups at 48 hours $(P<0.001,0.01$, or 0.05$)$.

The effects of 75 and $100 \mu \mathrm{g} / \mathrm{ml}$ of MP on LPS-induced HMC proliferation were significantly better than the effects observed in the 12 and $30 \mu \mathrm{g} / \mathrm{ml}$ MP treatment groups $(P<0.001$ or 0.01), particularly in the MP100 group. This finding indicated that the effect of MP on LPS-induced HMC proliferation was dose-dependent.

Similar to the effect of MP, the effect of US combined with MBs (US+MBs) on LPS-induced HMC proliferation was also dependent on the MB concentration. The cell proliferation rates were significantly decreased in the US+MB2 $\left(3.22 \times 10^{7}\right.$ bubbles/ml $)$ and US + MB3 $\left(8.05 \times 10^{7} \mathrm{bubbles} / \mathrm{ml}\right)$ groups at 24 hours $(P<0.001,0.01$, or 0.05$)$, and in the three US + MB treatment groups at 48 hours $(P<0.001,0.01$, or 0.05$)$, particularly in the US + MB2 and US + MB3 groups, which were significantly better than US alone $(P<0.01)$.

The combination of US, MBs at the $1.29 \times 10^{7} \mathrm{bubbles} / \mathrm{ml}$ concentration, and $12 \mu \mathrm{g} / \mathrm{ml}$ of MP (US+MB1+MP12) significantly enhanced the antiproliferative effect of MP because the HMC proliferation rate was significantly lower in the US+ MB1+MP12 group than in the MP12 or US + MB1 $\left(1.29 \times 10^{7}\right.$ bubbles/ml) group $(P<0.01)$.

Effect of Different Treatments on LPS-Induced HMC Apoptosis. As shown in Fig. 2, the treatments with MP, US, US combined with MBs, and US combined with MBs plus MP increased LPS-induced HMC apoptosis but to varying extents. Treatment with $10 \mu \mathrm{g} / \mathrm{ml}$ of LPS decreased the cell apoptosis rate compared with the control group at 24 and 48 hours, although the difference was not statistically significant $(P>$ 0.05 , Fig. 2). However, in comparison with the LPS treatment group, the cell apoptosis rate was increased in the MP treatment groups at 24 and 48 hours but only reached statistical significance in the MP75 $(75 \mu \mathrm{g} / \mathrm{ml}$ of MP) and MP100 $(100 \mu \mathrm{g} / \mathrm{ml}$ of MP) groups $(P<0.001$ or 0.01$)$. This was particularly the case in the MP100 group, which was also statistically significantly higher than both the MP12 $(12 \mu \mathrm{g} / \mathrm{ml}$ of MP) and MP30 (30 $\mu \mathrm{g} / \mathrm{ml}$ of MP) groups $(P<0.001$ or 0.01$)$ at 24 hours or than the MP12 group at 48 hours $(P<0.05)$. Otherwise, the cell apoptosis rates were higher at 48 hours than at 24 hours. The effect of MP on the LPS-induced HMC apoptosis rate occurred in a dose-dependent and timedependent manner.

Similar to the MP effect, US+MB increased HMC apoptosis in a dose-dependent and time-dependent manner. Among three US + MB treatment groups, the cell apoptosis rate was the highest in the US+MB3 group; the apoptosis rate in this group was significantly higher than the rate in LPS treatment group or the US or US+MB1 groups at 24 and 48 hours $(P<0.001,0.01$, or 0.05 ). US combined with MBs at the $1.29 \times 10^{7} \mathrm{bubbles} / \mathrm{ml}$ concentration and $12 \mu \mathrm{g} / \mathrm{ml}$ of MP significantly enhanced the effect of MP on HMC apoptosis $(P<0.001$ or 0.01$)$, resulting in a higher cell apoptosis rate than the US+MB1 group or the MP12 group, particularly at 24 hours after treatment.

TABLE 2

Primer sequences used for semiquantitative RT-PCR

\begin{tabular}{llr}
\hline \multicolumn{1}{c}{ Gene } & \multicolumn{1}{c}{ Forward Primer } & Reverse Primer \\
\hline NF- $\kappa$ B & 5'-AGGCTATCAGTCAGCGCATC-3' & 5'-CACTGTCACCTGGAAGCAGA-3' \\
TGF- $\beta 1$ & 5'-AGGACTGCGGATCTCTGTGT-3' & 5'-GGGCAAAGGAATAGTGCAGA-3' \\
GAPDH & 5'-CTCCTCCACCTTTGACGCTG-3' & 5'-TCCTCTTGTGCTCTTGCTGG-3' $^{\prime}$ \\
\hline
\end{tabular}




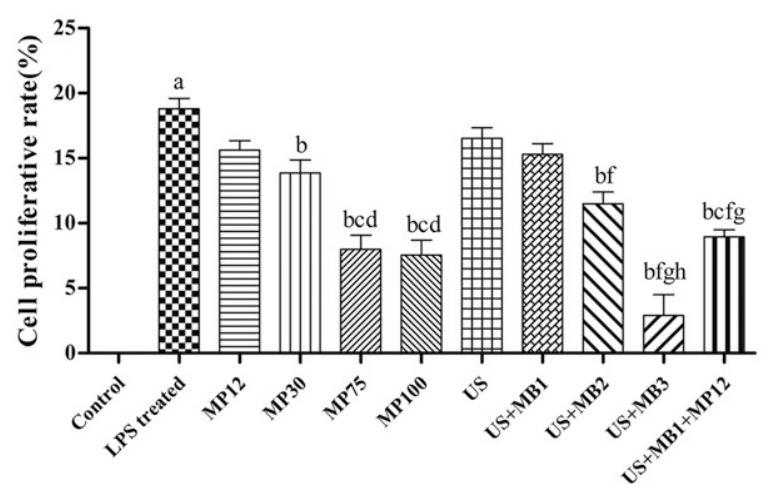

$24 \mathrm{~h}$

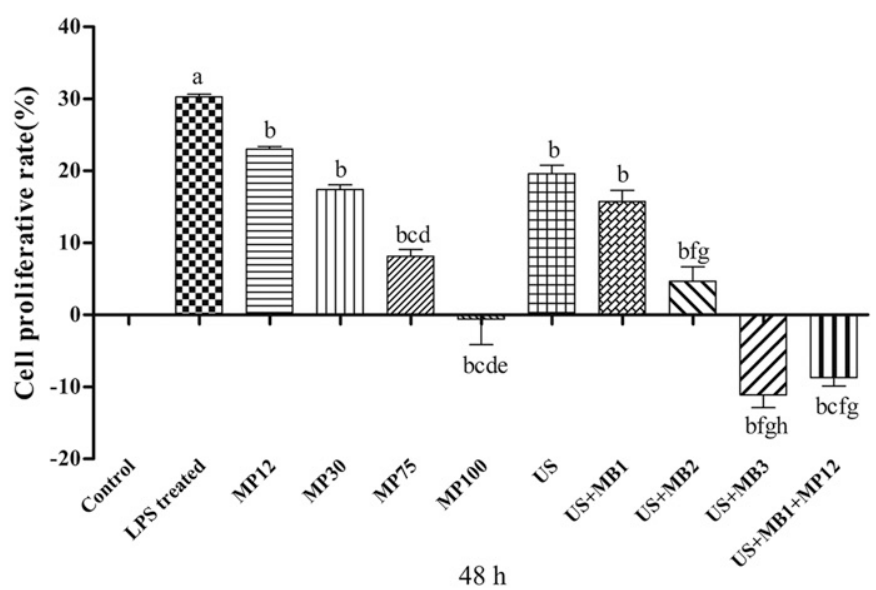

$48 \mathrm{~h}$

Fig. 1. Effects of different treatment on LPS-induced HMCs proliferative rate. HMCs were treated with $10 \mu \mathrm{g} / \mathrm{ml}$ of LPS alone (LPS treated group) or cotreated with US (US group), US combined with different MBs concentrations $\left(1.29 \times 10^{7}, 3.22 \times 10^{7}\right.$, or $8.05 \times 10^{7} \mathrm{bubbles} / \mathrm{ml}$; US+MB1, US+MB2, and US+MB3 groups, respectively), US combined with both MBs $\left(1.29 \times 10^{7}\right.$ bubbles/ml) and MP (12 $\left.\mu \mathrm{g} / \mathrm{ml}\right)$ (US+MB1+MP12 group), and different concentrations of MP alone (12, 30, 75, and $100 \mu \mathrm{g} / \mathrm{ml}$; MP12, MP30, MP75, and MP100 groups, respectively) for 24 or 48 hours. The effect on cell proliferation was measured by MTT methods. Cell proliferation rate $(\%)=\left(\right.$ Mean $\left.\mathrm{OD}_{\text {treatment group }}-\mathrm{Mean} \mathrm{OD}_{\text {control group }}\right) / \mathrm{Mean} \mathrm{OD}_{\text {control group }} \times 100 . \mathrm{Data}$ are expressed as mean \pm S.E.M., with the error bar representing the S.E.M. $(n=3$ per group). Statistical analysis was performed with ANOVA followed by Turkey's multiple comparison test. ${ }^{\mathrm{a}} P<0.001$, vs. control group. ${ }^{\mathrm{b}} P<0.001,0.01$, or 0.05 , vs. LPS-treated group. ${ }^{\mathrm{c}} P<0.001$ or 0.01 , vs. MP12 group. ${ }^{\mathrm{d}} P<0.001$ or 0.01 , vs. MP30 group. ${ }^{\mathrm{e}} P<0.05$, vs. MP75 group. ${ }^{\mathrm{f}} P<0.001$ or 0.05 , vs. US group. ${ }^{\mathrm{g}} P<0.001$ or 0.01 , vs. US+MB1 group. ${ }^{\text {h }} P<0.001$ or 0.01 , vs. US+MB2 group.

Effect of Different Treatments on LPS-Induced HMC NF- $\boldsymbol{k}$ B and TGF- $\beta 1$ Expression. The NF- $\kappa$ B and TGF- $\beta 1$ gene expression levels are shown in Fig. 3. The results showed that NF- $\kappa \mathrm{B}$ and TGF- $\beta 1$ gene expression was significantly increased at 24 or 48 hours after treatment with $10 \mu \mathrm{g} / \mathrm{ml}$ of LPS $(P<0.001,0.01$, or 0.05$)$. However, the gene expression levels were decreased at 24 and 48 hours after MP treatment. Compared with the LPS treatment group, the expression of the NF- $\kappa \mathrm{B}$ and TGF- $\beta 1$ genes was significantly inhibited by 75 or $100 \mu \mathrm{g} / \mathrm{ml}$ of MP at 24 hours $(P<0.01$ or 0.05$)$ and was additionally inhibited by $30 \mu \mathrm{g} / \mathrm{ml}$ of MP at 48 hours $(P<0.01$ or 0.05$)$; however, the differences between 24 and 48 hours were not statistically significant.
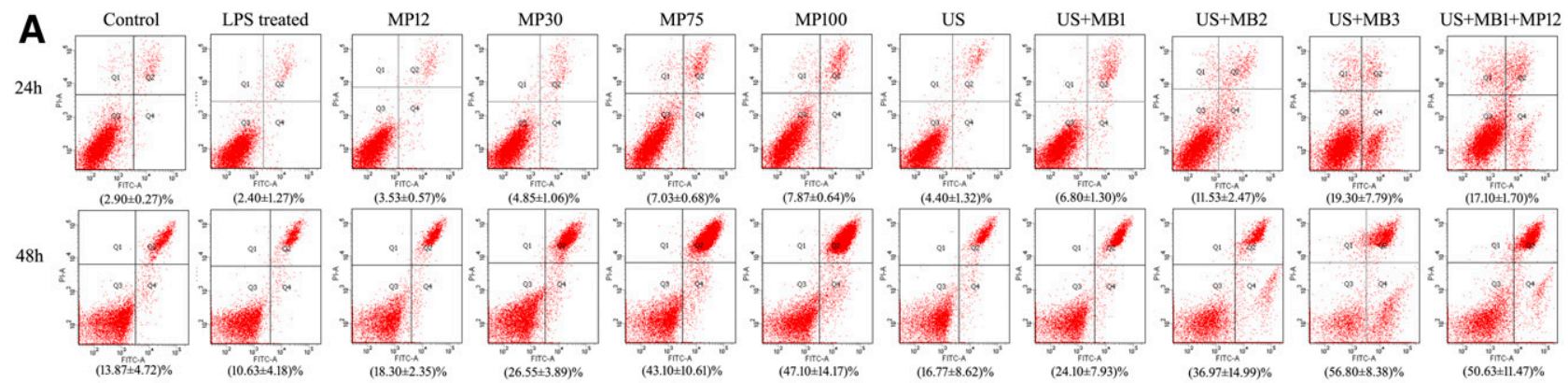

B
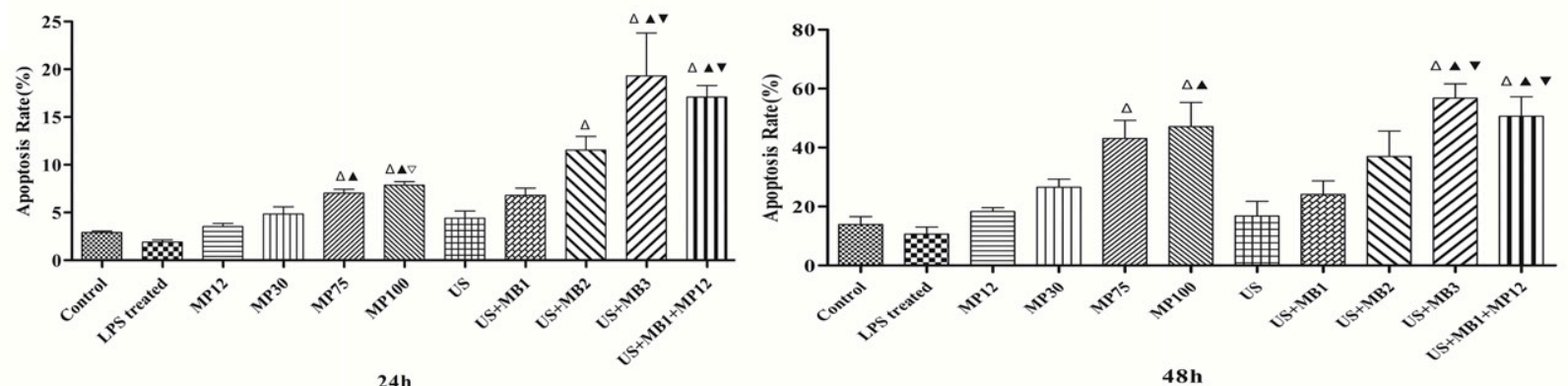

$48 \mathrm{~h}$

Fig. 2. HMC apoptosis rate at 24 and 48 hours after the different treatments. HMCs were treated as described in Fig. 1. Apoptosis was measured using a flow cytometer. Apoptosis rate $(\%)=($ Early apoptotic cells percentage + Late apoptotic cells percentage $) \times 100$. (A) Representative flow cytometric analysis diagram of the different groups at 24 and 48 hours. (B) Apoptosis rate analysis of the different groups at 24 and 48 hours. Data are expressed as mean \pm S.E.M. with error bar representing the S.E.M. $(n=3$ per group). Statistical analysis was performed with analysis of variance followed by Tukey's multiple comparison test. ${ }^{\Delta} P<0.001$ or 0.01 , vs. LPS treated group. ${ }^{\Delta} P<0.001,0.01$, or 0.05 , vs. MP12 group. ${ }^{\nabla} P<0.01$ or 0.05 , vs. MP30 group. $\nabla_{P}<0.001$ or 0.01, vs. US group or US+MB1 group. 
A

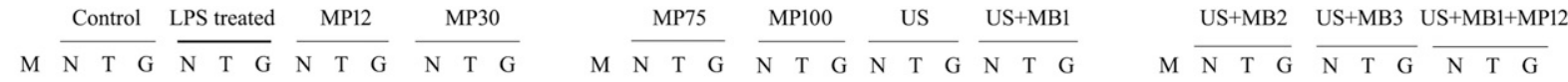

$24 \mathrm{~h}$

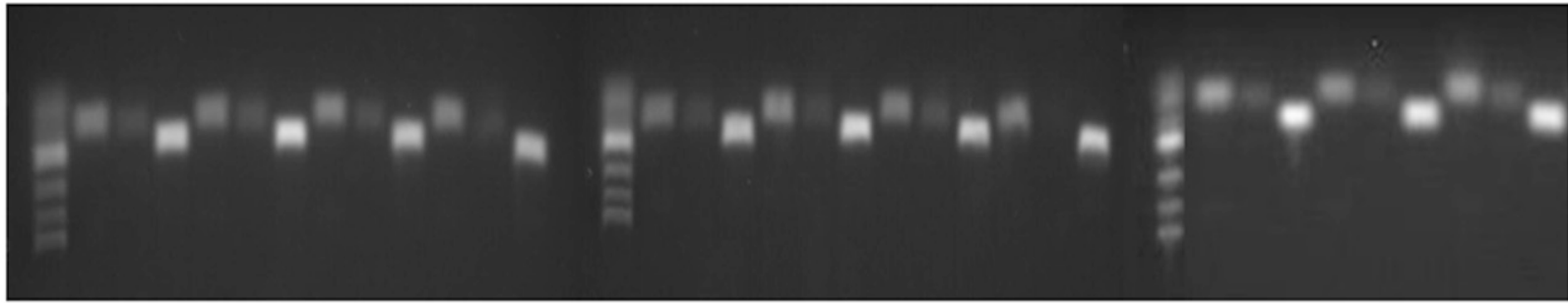

$48 \mathrm{~h}$

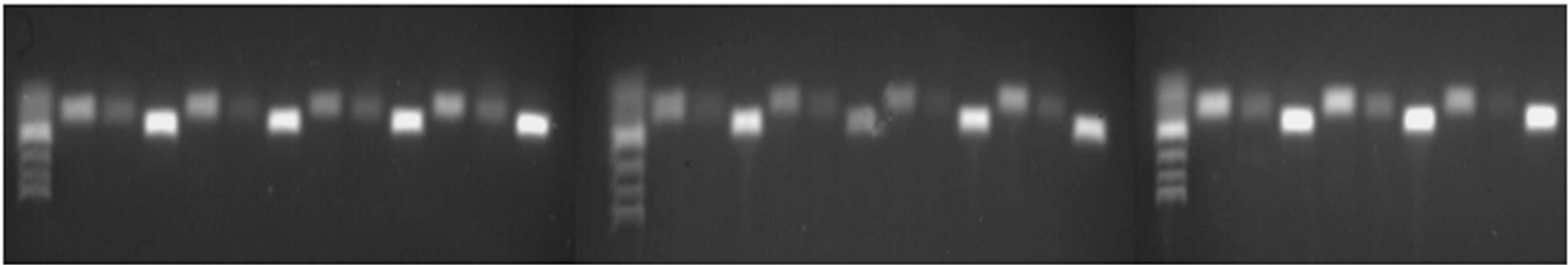

B

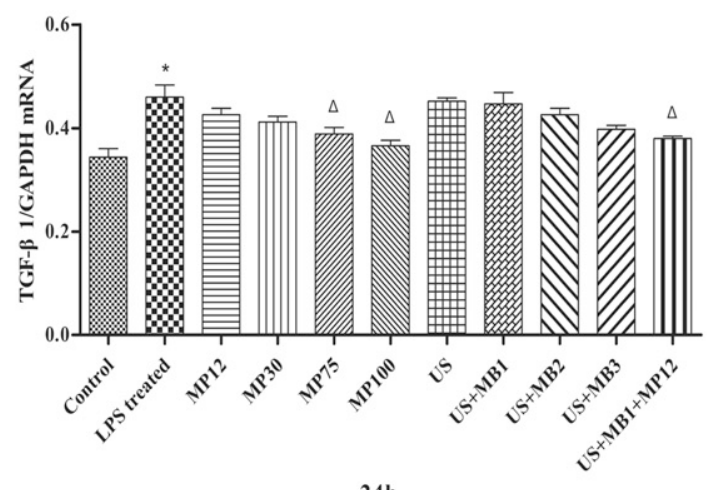

$24 h$

C

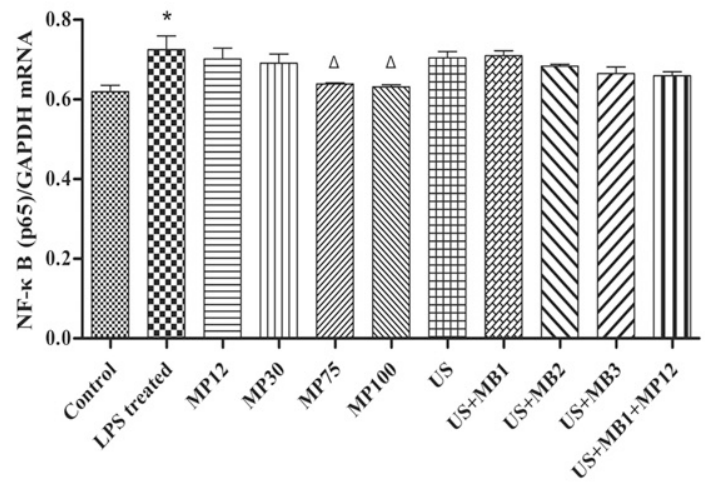

24h

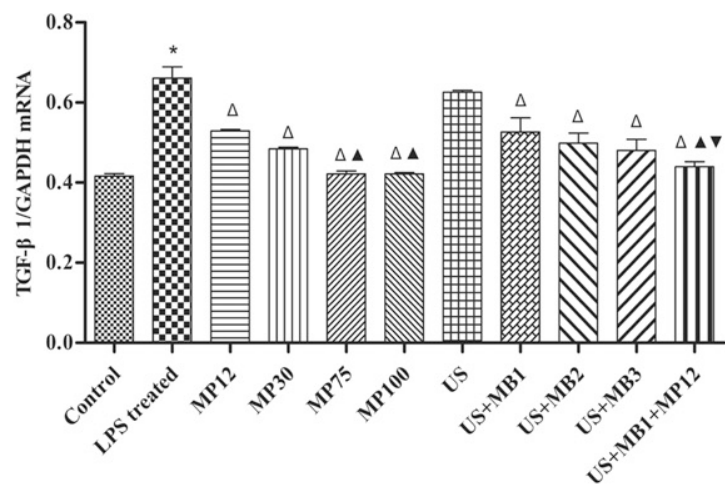

$48 \mathrm{~h}$

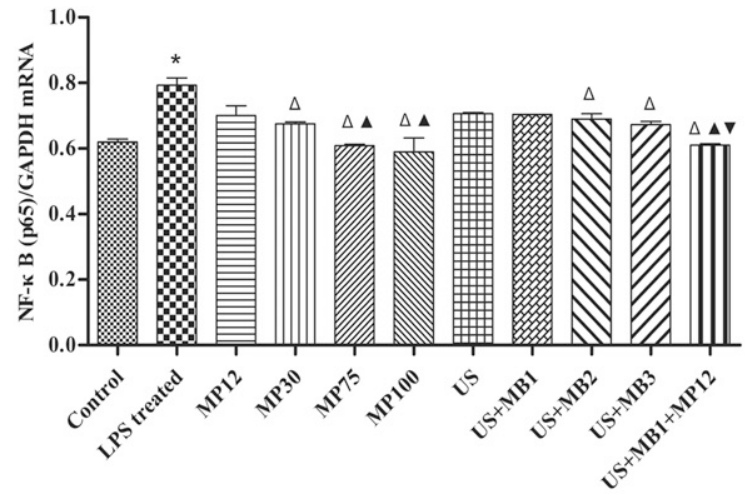

$48 \mathrm{~h}$

Fig. 3. TGF- $\beta 1$ and NF- $\kappa$ B mRNA expression in LPS-induced HMCs at 24 and 48 hours after the different treatments. HMCs were treated as described in Fig. 1. (A) Representative mRNA bands were quantified by RT-PCR analysis (G: GAPDH; M: marker; N: NF- $\kappa$ B; T:TGF- $\beta 1$ ). (B) Band density values of the TGF- $\beta 1$ mRNA were calculated as a ratio to GAPDH. (C) Band density values of the NF- $\kappa$ B mRNA were calculated as a ratio to GAPDH. Data are expressed as mean \pm S.E.M. with error bar representing the S.E.M. $(n=3$ per group). Statistical analysis was performed with analysis of variance followed by Tukey's multiple comparison test. ${ }^{*} P<0.001,0.01$, or 0.05 , vs. control group. ${ }^{\Delta} P<0.001,0.01$, or 0.05 , vs. LPS treated group. ${ }^{\Delta} P<0.05$, vs. MP12 group. ${ }^{\nabla} P<0.05$, vs. US+MB1 group.

The NF- $\kappa$ B and TGF- $\beta 1$ gene expression levels were significantly lower in the MP75 and MP100 groups than in the MP12 group at 48 hours $(P<0.01$ or 0.05$)$. These results indicated the MP could inhibit NF- $\kappa$ B and TGF- $\beta 1$ gene expression in a dose-dependent manner in LPS-induced proliferative HMCs.

After US or US + MB treatment, we observed that US combined with different MB concentrations also inhibited
$\mathrm{NF}-\kappa \mathrm{B}$ and TGF- $\beta 1$ gene expression, especially in the US+ MB2 and US+MB3 groups at 48 hours. Additionally, US combined with MBs at the $1.29 \times 10^{7}$ bubbles $/ m$ l concentration and $12 \mu \mathrm{g} / \mathrm{ml}$ of MP significantly decreased NF- $\kappa$ B and TGF- $\beta 1$ gene expression $(P<0.001$ or 0.01$)$ to a level lower than the US + MB1 group or the MP12 group, particularly at 48 hours after treatment. The NF- $\kappa$ B (p65) and TGF- $\beta 1$ 


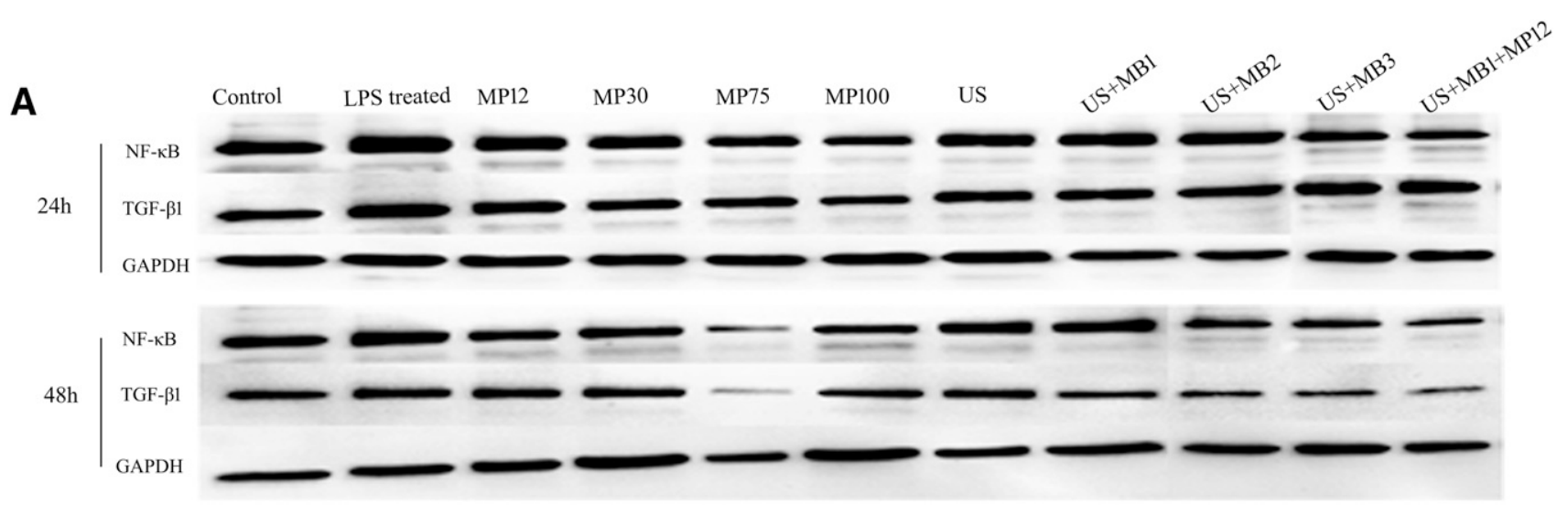

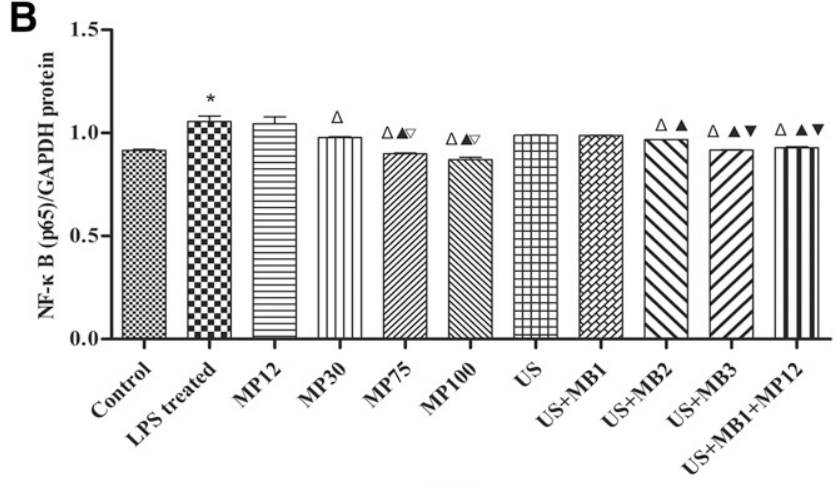

$24 \mathrm{~h}$

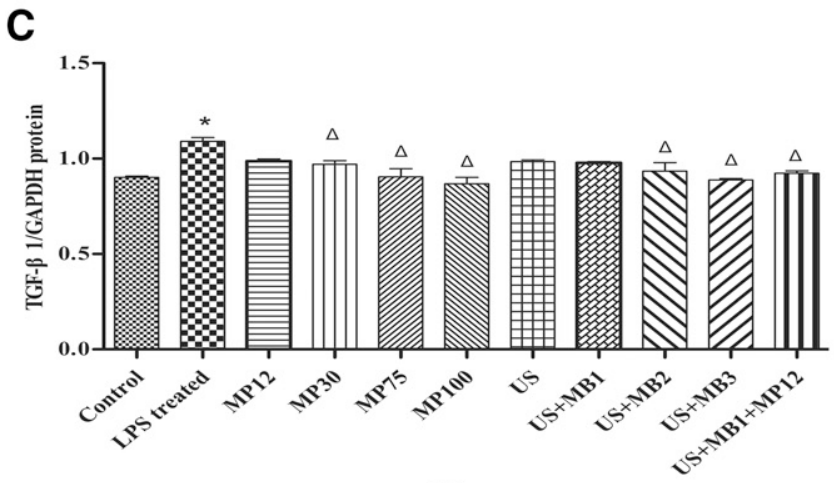

$24 \mathrm{~h}$

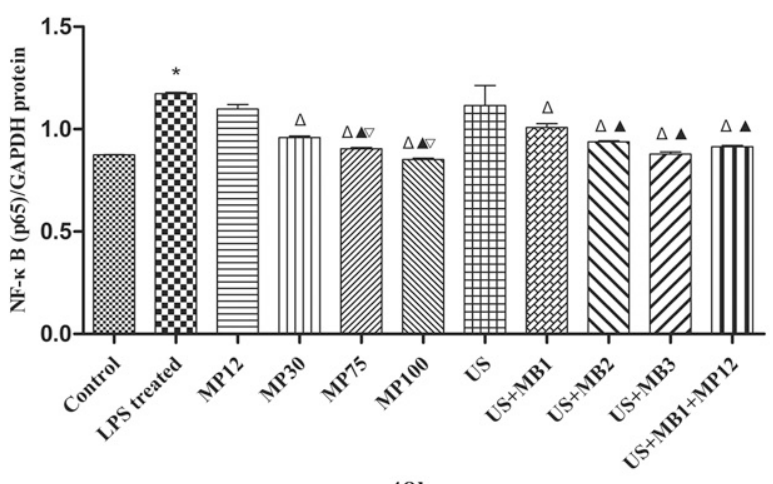

$48 \mathrm{~h}$

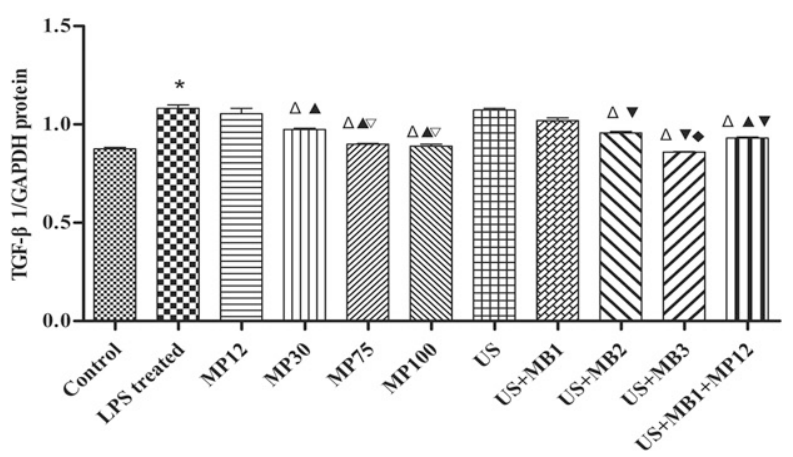

$48 \mathrm{~h}$

Fig. 4. NF- $\kappa$ B (p65) and TGF- $\beta 1$ protein expression in LPS-induced HMCs at 24 and 48 hours after the different treatments. HMCs were treated as described in Fig. 1. (A) Representative protein bands were quantified by Western blot analysis. (B) Band density values of the NF- $\kappa$ B (p65) protein were calculated as a ratio to GAPDH. (C) Band density values of the TGF- $\beta 1$ protein were calculated as a ratio to GAPDH. Data are expressed as mean \pm S.E. M. with error bar representing the S.E.M. ( $n=3$ per group). Statistical analysis was performed with analysis of variance followed by Tukey's multiple comparison test. ${ }^{*} P<0.001$, vs. control group. ${ }^{\Delta} P<0.001,0.01$, or 0.05 , vs. LPS treated group. ${ }^{\wedge} P<0.05$, vs. MP12 group. ${ }^{\nabla} P<0.001$ or 0.05 , vs. MP30 group. ${ }^{\nabla} P<0.05$, vs. US+MB1 group. ${ }^{\circ} P<0.001$, vs. US+MB2 group.

protein expression levels are shown in Fig. 4 and were consistent with the gene expression levels.

Intracellular Free MP Content. To explore the mechanism underlying the effect of US combined with MBs plus MP on LPS-induced proliferative HMC growth, the intracellular free MP content was measured using RP-HPLC. As shown in Table 3, after US + MB treatment, the intracellular free MP content was significantly increased in the US+ MB + MP12 group at 24 hours to a level higher than the increase observed in the MP12 group $(P<0.05)$; however, there was no significant difference between the contents at 48 hours.

\section{Discussion}

US contrast agents are thought to undergo oscillations that induce acoustic cavitation, thereby allowing an increase in the cell membrane, vessel, or blood-brain barrier permeability. The increased permeability enables drugs to cross the membrane, vessel, or blood-brain barrier to penetrate into cells, the extracellular space, or the brain and ultimately induce a therapeutic effect. Our study showed that conventional MBs could accumulate in inflamed kidneys due to phagocytosis by leukocytes in inflamed tissues after intravascular injection into animals or humans (Lindner et al., 2000; Deelman et al., 2010). 
TABLE 3

Intracellular free MP content after the treatment $(n=3)$

Data are expressed as mean \pm S.E.M.

\begin{tabular}{lcc}
\hline \multirow{2}{*}{ Groups } & \multicolumn{3}{c}{ MP Content } \\
\cline { 2 - 3 } & 24 Hours & 48 Hours \\
\hline & & $\mu g / m l$ \\
MP12 & $0.59 \pm 0.08$ & $0.32 \pm 0.05$ \\
US+MB+MP12 & $1.09 \pm 0.16^{a}$ & $0.47 \pm 0.08$ \\
\hline
\end{tabular}

${ }^{a} P<0.05$, versus MP12 group.

Because the kidneys receive approximately $25 \%$ of the cardiac output, a considerable number of MBs will enter the kidneys after intravenous or arterial injection. Thus, MBs are considered as a suitable vector for targeted drug or gene delivery to the diseased kidney (Deelman et al., 2010; Liang et al., 2010; Castle et al., 2013; Cao et al., 2016).

Generally, there are two alternative approaches to US-assisted drug delivery. First, the drug can be entrapped into or attached onto the MBs and administered into the vasculature to achieve US-triggered drug release from the MBs and localized tissue deposition in response to US treatment of the target zone. Second, the drug can be coadministered with the MBs (Escoffre and Bouakaz, 2016). Obviously, the second method is a very convenient approach due to the immediate availability of both the MBs and drug agents and thus is a straightforward continuation of the current use of clinically approved agents. This approach to drug delivery has been applied to treat diseases such as cardiovascular disease, central nervous system disease, and tumors (Ibsen et al., 2013; Aryal et al., 2014; de Saint Victor et al., 2014; Kotopoulis et al., 2014; Rychak and Klibanov, 2014), but it has been rarely applied to kidney disease.

RMC proliferation and ECM accumulation are universal morphologic manifestations of renal diseases and are involved in the pathogenesis of many renal diseases (Yang et al., 2016). LPS is an endotoxin that can exacerbate and accelerate nephritis in vivo and activates RMC in vitro to produce proinflammatory mediators, including interleukin-6, TGF$\beta 1, \mathrm{NF}-\kappa \mathrm{B}$, and tumor necrosis factor- $\alpha$ (TNF- $\alpha$ ) (Shui et al., 2007; Li et al., 2015). In our study the LPS-induced inflammatory responses in HMCs were used to explore the potential effect of the coadministration of MBs and MP mediated by US for glomerulonephritis therapy.

Numerous growth factors, cytokines, and signaling pathways are involved in the development of glomerulosclerosis. Among them, TGF- $\beta 1$ is the key profibrosis factor, with functions in cell growth, apoptosis, proliferation, and ECM production (Yang et al., 2016). TGF- $\beta 1$ can induce RMC proliferation and stimulate the synthesis and inhibit the degradation of the ECM, leading to the relentless progression of glomerulosclerosis (Herrera et al., 2011). Moreover, the $\mathrm{NF}-\kappa \mathrm{B}$ pathway is the major signaling pathway that controls inflammation-associated gene expression. Our study explored the effect of MB and MP coadministration mediated by US on LPS-induced HMC proliferation, apoptosis, and inflammatory mediator expression (NF- $\kappa \mathrm{B}$ and TGF- $\beta 1$ ) in vitro.

Similar to the results in previous studies (Xue et al., 2011; Li et al., 2015; Yang et al., 2016), we found that LPS markedly induced HMC proliferation, increased NF- $\kappa \mathrm{B}$ and TGF- $\beta 1$ mRNA and protein expression, and reduced HMC apoptosis. The LPS-induced inflammatory response was inhibited in a dose-dependent manner by treatment with different concentrations of MP alone, especially in the 75 and $100 \mu \mathrm{g} / \mathrm{ml} \mathrm{MP}$ groups compared with the 12 and $30 \mu \mathrm{g} / \mathrm{ml}$ MP groups. Thus, the inhibition of LPS-induced HMC proliferation by 75 and $100 \mu \mathrm{g} / \mathrm{ml}$ of MP might be associated with the increase in HMC apoptosis and the decrease in the expression of the NF- $\kappa \mathrm{B}$ and TGF- $\beta 1$ inflammatory mediators. When compared with MP12 alone or US alone or US+MB1 in the absence of MP, the antiinflammatory effect of MP at $12 \mu \mathrm{g} / \mathrm{ml}$ was significantly enhanced by cotreatment with $\mathrm{MBs}$ at the $1.29 \times 10^{7}$ bubbles/ml concentration under US exposure (US+MB1+ MP12), which was comparable to that of an $>8$-fold higher concentration of MP (100 $\mu \mathrm{g} / \mathrm{ml}$, MP100 alone).

Based on the current view of the US-assisted drug-delivery mechanism, we hypothesized that the MB administration successfully induced sonoporation by US, thereby promoting drug entry into the cells for local drug delivery. This hypothesis was confirmed by the intracellular free MP content, which was significantly increased at 24 hours after treatment with US combined with MBs and MP. Therefore, our data demonstrate that US combined with MBs could improve the anti-inflammatory effect of MP via US-assisted MB local drug delivery in LPS-induced HMC proliferation when coadministered.

Additionally, we observed that US alone or combined with $\mathrm{MBs}$ at the $1.29 \times 10^{7} \mathrm{bubbles} / \mathrm{ml}$ concentration in the absence of MP markedly reduced LPS-induced HMC proliferation at 48 hours and that US+MB1 inhibited LPSinduced NF- $\kappa \mathrm{B}$ and TGF- $\beta 1$ expression slightly better than US alone. Therefore, our data indicate that ultrasound in the presence of microbubbles can exhibit an anti-inflammatory effect on LPS-induced HMCs in vitro via a MP independent mechanism.

Microbubbles have been considered to act as the cavitation nuclei to enhance the biologic and chemical effects of US, such as cell membrane structure changes, cell cycle arrest, morphologic repression, and cellular apoptosis (Miller and Thomas, 1995; Feril et al., 2003; Schlicher et al., 2010; Zhong et al., 2011; Zhao et al., 2015b). Other studies have also reported that US combined with MBs reduced NF- $\kappa$ B expression and induced cellular apoptosis in tumor therapy (Fang et al., 2011; Ferlazzo et al., 2016). Our data from the present study support the view that HMC apoptosis is associated with the low level of NF- $\kappa \mathrm{B}$ in addition to the direct US cavitation mechanism. Thus, we assumed that the antiproliferative effect of US combined with MBs might be mediated by reducing $\mathrm{NF}-\kappa \mathrm{B}$ and TGF- $\beta 1$ expression and promoting HMC apoptosis, but this issue needs to be explored in the future. With these results taken together, we conclude that US combined with MBs could synergize the pharmacologic effect of MP via multiple mechanisms.

As mentioned before, some patients have a poor response to GCs treatment or are considered to be steroid dependent. The underlying reason for the failure to respond to steroids might involve several factors, such as genetic mutation, the GC receptor $\alpha(\mathrm{GR} \alpha)$ to GR $\beta$ ratio, histologic type, proteinuria degree, or renal function (Lewis-Tuffin and Cidlowski, 2006; KDIGO Work Group, 2012; Han et al., 2017). In the clinic, relapse is very common in these patients, at which point high-dose or long-term steroid therapy is often recommended. However, repeated and prolonged steroid therapy is 
associated with an excess of adverse events, some of which are dose related (Zhao et al., 2015a).

Previous studies confirmed that US combined with MBs could enhance the delivery of genes or drug to target areas of tissues including the kidney (Ibsen et al., 2013; Aryal et al., 2014; Huang et al., 2016; Wei et al., 2016). A recent study showed that CoQ10-liposome in combination with US and MBs could improve the protective effect of the drug on the damaged kidney, as seen in diabetic nephropathy (Yue et al., 2017). Therefore, our study provides evidence to suggest that US combined with MBs and MP is likely to be a superior strategy to deliver MP locally to an organ such as the kidney at a lower dose to achieve a comparable or even better antiinflammatory effect in vivo with a reduced risk of the adverse effects that are commonly seen with high doses of MP.

In conclusion, ours is the first study to explore the effect of US combined with MBs or cotreatment with MP on LPSinduced HMC proliferation, apoptosis, and NF- $\kappa \mathrm{B}$ and TGF$\beta 1$ expression in vitro. Our study demonstrated that certain parameters of US combined with MBs not only inhibited LPSinduced HMC proliferation, NF- $\kappa \mathrm{B}$ and TGF- $\beta 1$ expression, and induced cellular apoptosis, but it also synergized the pharmacologic effect of MP. The mechanism was associated with US-assisted MB local drug delivery and the antiinflammatory effect induced by US combined with MBs.

These results provide insight into the anti-inflammatory effect induced by US combined with MBs and the initial mechanisms, which can be used as a basis for therapeutic purposes. However, there are some issues to examine in future research, including the exact dosage of MP and its efficacy in in vivo therapy for nephritis, the influence this novel approach has on the response to GC treatment, the anti-inflammation mechanism of US combined with MBs, and whether the same effects are evident when other US parameters are used.

\section{Acknowledgments}

The authors thank Yun-Feng Ma and Shao-Li Chen for help with the experiment.

\section{Authorship Contributions}

Participated in research design: Sun, Xu, Ai, Wang, Ren.

Conducted experiments: Sun, Tian, Fu, Wang, Zhang, Si.

Performed data analysis: Sun, Ren.

Wrote or contributed to the writing of the manuscript: Chen, Chai, Cooper, Ren.

\section{References}

Anderson CD, Moisyadi S, Avelar A, Walton CB, and Shohet RV (2016) Ultrasoundtargeted hepatic delivery of factor IX in hemophiliac mice. Gene Ther 23:510-519. Aryal M, Arvanitis CD, Alexander PM, and McDannold N (2014) Ultrasoundmediated blood-brain barrier disruption for targeted drug delivery in the central nervous system. Adv Drug Deliv Rev 72:94-109.

Cao WJ, Matkar PN, Chen HH, Mofid A, and Leong-Poi H (2016) Microbubbles and ultrasound: therapeutic applications in diabetic nephropathy. Adv Exp Med Biol 880:309-330.

Castle J, Butts M, Healey A, Kent K, Marino M, and Feinstein SB (2013) Ultrasoundmediated targeted drug delivery: recent success and remaining challenges. Am J Physiol Heart Circ Physiol 304:H350-H357.

Deelman LE, Declèves AE, Rychak JJ, and Sharma K (2010) Targeted renal therapies through microbubbles and ultrasound. Adv Drug Deliv Rev 62:1369-1377.

de Saint Victor M, Crake C, Coussios CC, and Stride E (2014) Properties, characteristics and applications of microbubbles for sonothrombolysis. Expert Opin Drug Deliv 11:187-209.

Escoffre J-M and Bouakaz A, editors (2016) Therapeutic Ultrasound, Springer, New York.

Fang LH, Jiang Z, and Qian ML (2011) Study of low-frequency ultrasound combined with microbubble agent on apoptosis of human hepatocellular carcinoma cells. Linchuang Zhongliuxue Zazhi 16:510-513.

Feril LB, Jr, Kondo T, Zhao QL, Ogawa R, Tachibana K, Kudo N, Fujimoto S, and Nakamura S (2003) Enhancement of ultrasound-induced apoptosis and cell lysis by echo-contrast agents. Ultrasound Med Biol 29:331-337.
Ferlazzo N, Cirmi S, Russo M, Trapasso E, Ursino MR, Lombardo GE, Gangemi S, Calapai G, and Navarra M (2016) NF- $\kappa$ B mediates the antiproliferative and proapoptotic effects of bergamot juice in HepG2 cells. Life Sci 146:81-91.

Han SS, Xu YQ, Lu Y, Gu XC, and Wang Y (2017) A PRISMA-compliant metaanalysis of MDR1 polymorphisms and idiopathic nephrotic syndrome: susceptibility and steroid responsiveness. Medicine (Baltimore) 96:e7191.

Herrera GA, Turbat-Herrera EA, and Teng J (2011) Mesangial homeostasis and pathobiology: their role in health and disease. Contrib Nephrol 169:6-22.

Huang H, Li X, Zheng S, Chen Y, Chen C, Wang J, Tong H, Zhou L, Yang J, and Zeng C (2016) Downregulation of renal G protein-coupled receptor kinase type 4 expression via ultrasound-targeted microbubble destruction lowers blood pressure in spontaneously hypertensive rats. J Am Heart Assoc 5e004028.

Ibsen S, Schutt CE, and Esener S (2013) Microbubble-mediated ultrasound therapy: a review of its potential in cancer treatment. Drug Des Devel Ther 7:375-388.

Imig JD and Ryan MJ (2013) Immune and inflammatory role in renal disease. Compr Physiol 3:957-976.

Jamba A, Kondo S, Urushihara M, Nagai T, Kim-Kaneyama JR, Miyazaki A, and Kagami S (2015) Hydrogen peroxide-inducible clone-5 regulates mesangial cell proliferation in proliferative glomerulonephritis in mice. PLoS One 10: e0122773.

Kidney Disease: Improving Global Outcomes (KDIGO) Work Group (2012) Chapter 4: steroid-resistant nephrotic syndrome in children. Kidney Int Suppl (2011) 2: 172-176 DOI: 10.1038/kisup.2012.17.

Kotopoulis S, Delalande A, Popa M, Mamaeva V, Dimcevski G, Gilja OH, Postema M, Gjertsen BT, and McCormack E (2014) Sonoporation-enhanced chemotherapy significantly reduces primary tumour burden in an orthotopic pancreatic cancer xenograft. Mol Imaging Biol 16:53-62.

Lewis-Tuffin LJ and Cidlowski JA (2006) The physiology of human glucocorticoid receptor beta (hGR $\beta)$ and glucocorticoid resistance. Ann N Y Acad Sci 1069:1-9.

Li G, Fu J, Zhao Y, Ji K, Luan T, and Zang B (2015) Alpha-lipoic acid exerts antiinflammatory effects on lipopolysaccharide-stimulated rat mesangial cells via inhibition of nuclear factor kappa B (NF- $\kappa$ B) signaling pathway. Inflammation 38 : $510-519$

Li H, Qian J, Yao C, Wan C, and Li F (2016) Combined ultrasound-targeted microbubble destruction and polyethylenimine-mediated plasmid DNA delivery to the rat retina: enhanced efficiency and accelerated expression. J Gene Med 18 47-56.

Liang HD, Tang J, and Halliwell M (2010) Sonoporation, drug delivery, and gene therapy. Proc Inst Mech Eng H 224:343-361.

Lindner JR, Song J, Xu F, Klibanov AL, Singbartl K, Ley K, and Kaul S (2000) Noninvasive ultrasound imaging of inflammation using microbubbles targeted to activated leukocytes. Circulation 102:2745-2750.

MacHardy N, Miles PV, Massengill SF, Smoyer WE, Mahan JD, Greenbaum L, Massie S, Yao L, Nagaraj S, Lin JJ, et al. (2009) Management patterns of childhood-onset nephrotic syndrome. Pediatr Nephrol 24:2193-2201.

Miller DL and Thomas RM (1995) Ultrasound contrast agents nucleate inertial cavitation in vitro. Ultrasound Med Biol 21:1059-1065.

Ponticelli C, Villa M, Banfi G, Cesana B, Pozzi C, Pani A, Passerini P, Farina M, Grassi C, and Baroli A (1999) Can prolonged treatment improve the prognosis in adults with focal segmental glomerulosclerosis? Am J Kidney Dis 34:618-625.

Radeke HH and Resch K (1992) The inflammatory function of renal glomerular mesangial cells and their interaction with the cellular immune system. Clin Investig 70:825-842.

Ren X, Wang Y, Wang Y, Chen H, Chen L, Liu Y, and Xue C (2015) Thrombolytic therapy with rt-PA and transcranial color Doppler ultrasound (TCCS) combined with microbubbles for embolic thrombus. Thromb Res 136:1027-1032.

Rychak JJ and Klibanov AL (2014) Nucleic acid delivery with microbubbles and ultrasound. Adv Drug Deliv Rev 72:82-93.

Sánchez-López E, Rodriguez-Vita J, Cartier C, Rupérez M, Esteban V, Carvajal G, Rodrígues-Díez R, Plaza JJ, Egido J, and Ruiz-Ortega M (2008) Inhibitory effect of interleukin- $1 \beta$ on angiotensin II-induced connective tissue growth factor and type IV collagen production in cultured mesangial cells. Am J Physiol Renal Physiol 294:F149-F160.

Schlicher RK, Hutcheson JD, Radhakrishna H, Apkarian RP, and Prausnitz MR (2010) Changes in cell morphology due to plasma membrane wounding by acoustic cavitation. Ultrasound Med Biol 36:677-692.

Schöcklmann HO, Lang S, and Sterzel RB (1999) Regulation of mesangial cell proliferation. Kidney Int 56:1199-1207.

Shui HA, Ka SM, Wu WM, Lin YF, Hou YC, Su LC, and Chen A (2007) LPS-evoked IL-18 expression in mesangial cells plays a role in accelerating lupus nephritis. Rheumatology (Oxford) 46:1277-1284.

Wang YU, Chen YN, Zhang W, Yang YU, Bai WK, Shen E, and Hu B (2016) Upregulation of ULK1 expression in PC-3 cells following tumor protein P53 transfection by sonoporation. Oncol Lett 11:699-704.

Wei S, Xu C, Rychak JJ, Luong A, Sun Y, Yang Z, Li M, Liu C, Fu N, and Yang B (2016) Short hairpin RNA knockdown of connective tissue growth factor by ultrasound-targeted microbubble destruction improves renal fibrosis. Ultrasound Med Biol 42:2926-2937.

Wu F, Zhang W, Li L, Zheng F, Shao X, Zhou J, and Li H (2011) Inhibitory effects of honokiol on lipopolysaccharide-induced cellular responses and signaling events in human renal mesangial cells. Eur $J$ Pharmacol 654:117-121.

Xue Y, Tie CR, Li J, Tian T, and Li QX (2011) Ligustrazine inhibits lipopolysaccharide-induced proliferation by affecting P27, Bcl-2 expression in rat mesangial cells. Eur J Pharmacol 665:8-12.

Yang L, Sun X, Zhan Y, Liu H, Wen Y, Mao H, Dong XI, and Li P (2016) Yi Qi Qing Re Gao-containing serum inhibits lipopolysaccharide-induced rat mesangial cell proliferation by suppressing the Wnt pathway and TGF- $\beta 1$ expression. Exp Ther Med 11:1410-1416

Yue T, Xu HL, Chen PP, Zheng L, Huang Q, Sheng WS, Zhuang YD, Jiao LZ, Chi TT, ZhuGe DL, et al. (2017) Combination of coenzyme Q10-loaded liposomes with 
ultrasound targeted microbubbles destruction (UTMD) for early theranostics of diabetic nephropathy. Int $J$ Pharm 528:664-674.

Zhang X, Han X, Tang Y, Wu Y, Qu B, and Shen N (2015) miR-744 enhances type I interferon signaling pathway by targeting PTP1B in primary human renal mesangial cells. Sci Rep 5:12987.

Zhao L, Cheng J, Zhou J, Wu C, and Chen J (2015a) Enhanced steroid therapy in adult minimal change nephrotic syndrome: a systematic review and meta-analysis. Intern Med 54:2101-2108.

Zhao L, Feng Y, Shi A, Zong Y, and Wan M (2015b) Apoptosis induced by microbubble-assisted acoustic cavitation in K562 cells: the predominant role of the cyclosporin A-dependent mitochondrial permeability transition pore. Ultrasound Med Biol 41:2755-2764.
Zhong W, Sit WH, Wan JM, and Yu AC (2011) Sonoporation induces apoptosis and cell cycle arrest in human promyelocytic leukemia cells. Ultrasound Med Biol 37: $2149-2159$

Zhu X, Guo J, He C, Geng H, Yu G, Li J, Zheng H, Ji X, and Yan F (2016) Ultrasound triggered image-guided drug delivery to inhibit vascular reconstruction via paclitaxel-loaded microbubbles. Sci Rep 6:21683.

Address correspondence to: Dr. Shu-Ting Ren, Department of Pathology, School of Basic Medical Sciences, Xi'an Jiaotong University Health Science Center, Yanta West Road No. 76, Xi'an 710061, People's Republic of China. E-mail: rst@mail.xjtu.edu.cn 